\title{
Generation of Continuous-Time Two Integrator Loop OTA Filter Structures
}

\author{
EDGAR SÁNCHEZ-SINENCIO, SENIOR MEMBER, IEEE, RANDALL L. GEIGER, \\ SENIOR MEMBER, IEEE, AND H. NEVAREZ-LOZANO
}

\begin{abstract}
Generation of two integer loop filter structures using operational $T$ ransconductance $A$ mplifiers (OTA's) and Capicitors (TAC) is presented. A direct block diagram approach is employed to generate the TAC filter structures. The basic filter building blocks consist of a lossless integrator, lossy integrators and three types of summers. They are implemented using OTA's as the active components. The resulting TAC second-order filter structures, some previously reported and other new structures, are orderly generated. It is shown that the selection of the best structure depends upon the particular application or design specifications. The generated TAC biquadratic structures are very suitable for monolithic filters since typically only OTA's and two capacitors are needed. A TAC filter structure homologous to the conventional KHN op amp filter structure is presented. Programmability of $\omega_{p}, Q_{p}$ and voltage gain is considered.
\end{abstract}

\section{INTRODUCTION}

$\mathbf{R}$ ECENTLY, several research groups have been searching for different alternatives to design continuous-time active filters [1]-[10] by making the time constant voltage- or current-dependent. In this paper, we further discuss filter structures using operational transconductance amplifiers (OTA's) as the active components in continuous-time active filters [19]-[23]. Moreover, the transconductance gain $g_{m}$ of the OTA is used as a design parameter in the same way as the R's are used in conventional active-RC filters. Many existing drawbacks in commercial OTAs [11]-[15] are overcome by a CMOS OTA [16]-[18] structure capable of handling larger input voltage differences. Thus the main advantages in using $g_{m}$ as a design parameter are that: i) $g_{m}$ can be varied or programmed by an external controlling current or voltage; this flexibility permits the designer to meet a variety of design specifications, and ii) a good input voltage swing is possible for CMOS OTA's.

Let us introduce the basic operations of the OTA circuits. The OTA ideally is a DVCCS with infinite output impedance and zero-input currents. Fig. 1 shows a summer operation circuit. The output voltage is given by

$$
V_{o}=\sum_{i=1}^{n} I_{o i} Z_{L}=\sum_{i=1}^{n} g_{m i}\left(V_{i a}-V_{i b}\right) Z_{L}
$$

Equation (1) involves three types of summers. The first summer occurs at the input terminals of the OTA. This

Manuscript received December 18, 1986; revised August 17, 1987. This paper was recommended by Associate Editor K. W. Martin.

The authors are with the Department of Electrical Engineering, Texas A\&M University, College Station, TX 77843.

IEEE Log Number 8821670 .

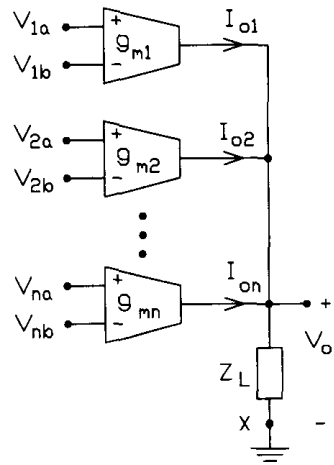

Fig. 1. A summer operation.

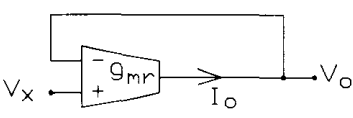

(a)

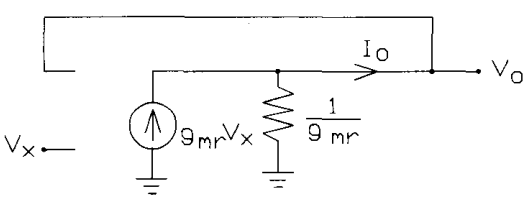

(b)

Fig. 2. Equivalent resistor. (a) Circuit. (b) Small signal circuit.

summer denominated $\Sigma_{o}$ is the difference between the input signals $V_{i a}$ and $V_{i b}$. The second summer occurs when the load is a capacitor $C$ (i.e., $Z_{L}=1 / s C$ ), the summation of the currents $I_{o i}$ is integrated and transformed into a voltage. This summer is denoted as $Z_{C}$. The third summer occurs when the load is an equivalent resistor (i.e., $Z_{L}=$ $\left.1 / g_{m r}\right)$. This summer is named $\sum_{r}$. The equivalent resistor [15] is shown in Fig. 2(a). Note from Fig. 2(b) that assuming an ideal OTA, analysis yields

$$
V_{0}=\frac{g_{m r} V_{x}}{g_{m r}}=V_{x}
$$

This implies that a unity weight factor ${ }^{1}$ is obtained be-

${ }^{1}$ For the injection of signal $V_{x}$ it is assumed an ideal voltage source. 


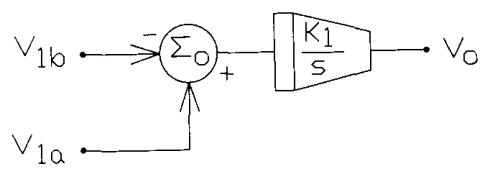

Fig. 3. Lossless integrator; block diagram representation.

tween the input signal $V_{x}$ and the output $V_{o}$. Furthermore, for the particular case when $V_{x}=0$, the equivalent grounded resistor yields

$$
R_{\mathrm{eq}}=\left.\frac{V_{o}}{-I_{o}}\right|_{V_{x}=0}=\frac{1}{g_{m r}} .
$$

Another basic circuit, the lossy integrator, will be discussed in the next section. This consists, in general, of either a lossless integrator with a feedback signal at the input of the lossless integrator, or a lossless integrator with an equivalent resistor load implemented with an additional OTA.

It should be expected from the three types of summers and different lossy integrators that a variety of two integrator loop filter structures can be generated. The same comment applies to the implementation of zeros since the number and type of summers available will determine the flexibility of the structure.

\section{INTEGRATOR AND SUMmer STRUCTURES}

To facilitate an orderly generation of two integrator loops we will introduce a block diagram representation of the basic operations, that is, for the three types of summers and the lossy integrators.

A basic OTA lossless integrator consists of an OTA and one capacitors. For instance consider Fig. 1 with only the OTA 1 and the load is a capacitor of value $C$. Observe that this integrator can be differential, positive, or negative by choosing or grounding the appropriate terminals. Note that the integrators discussed in this paper have a high output impedance. This fact is emphasized by the truncated triangle representing the integrator in Fig. 3, where $K_{1}=g_{m 1} / C$.

The transfer function, in general, of a lossy integrator (first-order low-pass filter) is

$$
H(s)=\frac{V_{o}(s)}{V_{\text {in }}(s)}=\frac{K_{2}}{s+K_{2} A_{1}}
$$

where $K_{2} A_{1}$ is the $3-\mathrm{dB}$ cutoff frequency, $\omega_{3 \mathrm{~dB}}$, and $1 / A_{1}$ is the low-frequency gain. Let us discuss, initially, the integrators from the point of view of block diagrams. The lossy integrator structures with high output impedance consist basically of a lossless equivalent integrator $K_{2} / \mathrm{s}$ with a local feedback component $A_{1} K_{2} / s$ and present two summers $\Sigma_{o}$ and $\Sigma_{C}$. This is illustrated in Fig. 4(a). The other lossy integrator type is shown in Fig. 4(b). It consists of a global feedback component $A_{1}$. Note that the feedback signal and the input are algebraically added through a summer $\Sigma_{o}$. Next, we discuss the OTA lossy integrator implementations. Fig. 5(a) shows the implementation of a

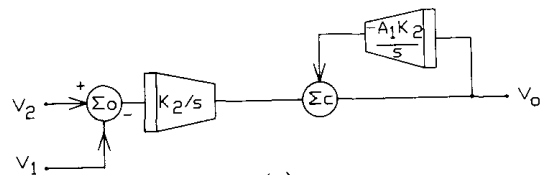

(a)

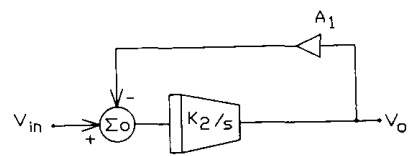

(b)

Fig. 4. Lossy integrator block diagrams; (a) Self-loop feedback component, (b) Lossless integrator plus a feedback component.

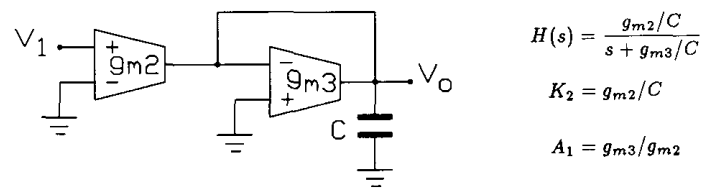

(a)

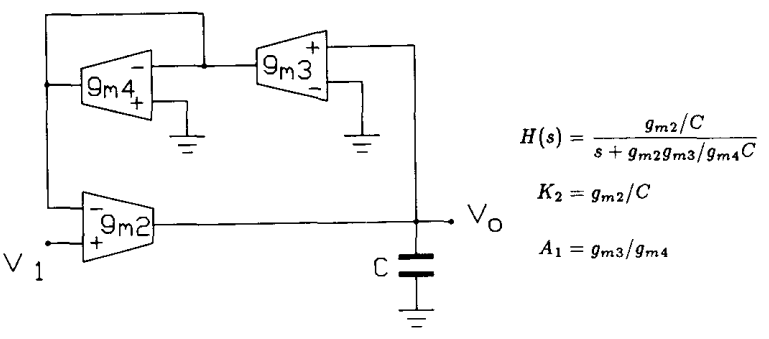

(b)

Fig. 5. Lossy integrator implementations. (a) Lossless integrator with a self-loop feedback component (b) Lossless integrator with variable feedback component.

lossy integrator that can be represented by Fig. 4(b). The $g_{m_{3}}-g_{m_{4}}$ combination for the particular case when $A_{1}=1$ can be simply replaced by a short circuit.

\section{Two INTEgrator LOOP TAC STRUCtURES}

The two initiator loop structures consist of a lossless integrator and a lossy integrator. Using the two block diagram representations of the lossy integrator of Fig. 4, we can obtain the corresponding fundamental two integrator loops of Fig. 6(a) and 6(b). The characteristic equation is identical for both structures and is given by

$$
D(s)=s^{2}+K_{2} A_{1} s+K_{1} K_{2} A_{0}
$$

where

$$
K_{2} A_{1}=\frac{\omega_{p}}{Q_{p}} \quad \text { and } \quad K_{1} K_{2} A_{0}=\omega_{p}^{2}
$$

thus $\omega_{p}$ and $Q_{p}$, the pole frequency and the pole quality factor, respectively, become

$$
\begin{aligned}
& \omega_{p}=\left[K_{1} K_{2} A_{0}\right]^{1 / 2} \\
& Q_{p}=\frac{1}{A_{1}}\left[K_{1} A_{0} / K_{2}\right]^{1 / 2} .
\end{aligned}
$$




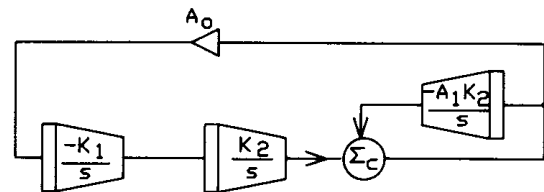

(a)

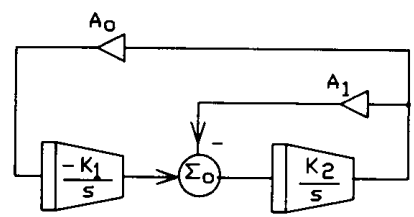

(b)

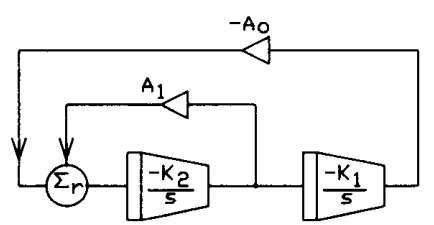

(c)

Fig. 6. Two integrator loops. (a) Cascade of one lossless and one lossy integrators. (b) Cascade of two lossless integrators. (c) Two integrator loops with a single feedback summer.

From (6) and (7) it can be observed that $\omega_{p}$ and $Q_{p}$ can be independently controlled, if $K_{1}, K_{2}, A_{0}$, and $A_{1}$ can be adjusted independently. Another feasible two integrator loop can be obtained by transposing the block diagram of Fig. 6(b) which is shown in Fig. 6(c). The characteristic equation is ideally given again by (5). However, observe that in Fig. 6(b) the output of the second integrator is feedback to the input of each integrator through $\sum_{o}$ summers while in Fig. 6(c) the output of each integrator is feedback to the input of one integrator through a $\Sigma_{r}$ summer. The main differences between these structures will be in the summer types and their corresponding injection points for the implementation of transmission zeros. For the following analysis, only the dead network of these filters will be considered whereas methods of controlling zeros will be discussed in Section IV.

Let us now consider the circuit implementations of structures of Fig. 6. The circuit derived from the block diagram structure of Fig. 6(a) is shown in Fig. 7. One particular case of this structure is when $A_{0}=1$, this implies that the combination of OTA's 4 and 5 can be replaced by a short circuit. The circuit implementation of the block diagram of Fig. 6(b), is shown in Fig. 8. There are three interesting cases for this structure: i) The general case $A_{0} \neq 1, A_{1} \neq 1$; ii) $A_{0}=1, A_{1} \neq 1$; and iii) $A_{0}=A_{1}=1$. Recall $^{2}$ that when the scaling factors $A_{0}$ or $A_{1}$ become one, they can simply be replaced by a short circuit. The circuit implementation of Fig 6(c) is shown in Fig. 9, it contains three available signal nodes, five OTA's and one weighted

\footnotetext{
${ }^{2} A_{0} \neq 1, A_{1}=1$ is another case, however these features are very similar to Topology 2 of Fig. 7 and involves more OTA's.
}

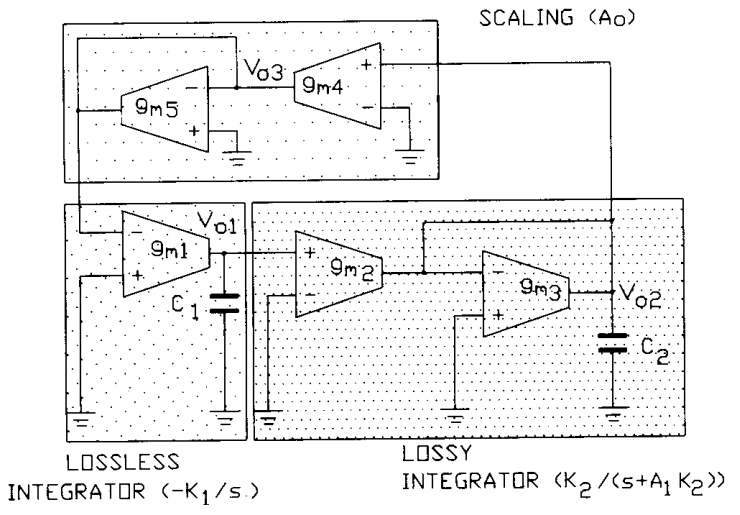

Fig. 7. Circuit implementation of Fig. 6(a).

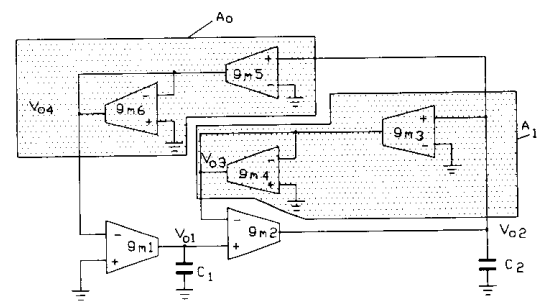

Fig. 8. Circuit implementation of Fig. 6(b).

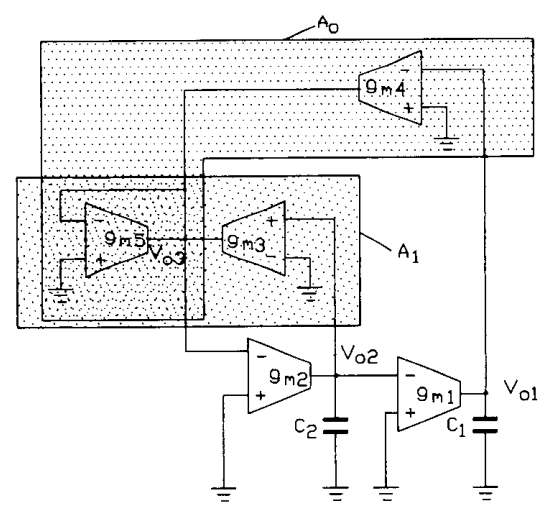

Fig. 9. Circuit implementation of Fig. 6(c).

TABLE I

$\omega_{p}, Q_{p}$ EXPRESSIONS For LOOP STRUCTURES OF Figs. 7, 8 AND 9

\begin{tabular}{|c|c|c|c|c|c|}
\hline Topology & $A_{0}$ & $A_{1}$ & $\omega_{p}^{2}$ & $Q_{p}$ & $\omega_{\mathrm{p}} / Q_{\mathrm{p}}$ \\
\hline 1. Fig. 7 & $\frac{g_{m_{1}}}{g_{m_{s}}}$ & $\frac{g_{m_{3}}}{g_{m_{2}}}$ & $\frac{g_{m_{1}} g_{m_{3}} g_{m_{3}}}{C_{1} C_{2} g_{m_{3}}}$ & $\frac{1}{g_{m_{3}}}\left[\frac{g_{m_{2}} g_{m_{3}} g_{m_{4}} C_{2}}{g_{m_{5}} C_{1}}\right]^{\frac{1}{2}}$ & $\frac{g_{m_{3}}}{C_{2}}$ \\
\hline 2. Fig. 7 & 1 & $\frac{g_{m_{3}}}{g_{m_{3}}}$ & $\frac{g_{m_{1}} g_{m_{2}}}{C_{1} C_{2}}$ & $\frac{1}{g_{m_{3}}}\left[\frac{g_{m_{2}} g_{m_{2}} C_{2}}{C_{1}}\right]^{\frac{1}{2}}$ & $\frac{g_{m 3}}{C_{2}}$ \\
\hline 3. Fig. 8 & $\frac{g_{m_{B}}}{g_{m_{s}}}$ & $\frac{g_{m_{3}}}{g_{m_{4}}}$ & $\frac{g_{m_{1}} g_{m_{3}} g_{m_{s}}}{C_{1} C_{2} g_{m_{s}}}$ & $\frac{g_{m_{4}}}{g_{m_{3}}}\left[\frac{g_{m_{1}} C_{2} g_{m_{3}}}{g_{m_{3}} C_{1} g_{m_{8}}}\right]^{\frac{1}{2}}$ & $\frac{g_{m_{2}} g_{m_{3}}}{C_{2} g_{m_{4}}}$ \\
\hline 4. Fig. 8 & 1 & $\frac{g_{m_{s}}}{g_{m_{4}}}$ & $\frac{g_{m_{1}} g_{m_{2}}}{C_{1} C_{2}}$ & $\frac{g_{m_{1}}}{g_{m_{3}}}\left[\frac{g_{m_{1}} C_{2}}{g_{m_{2}} C_{1}}\right]^{\frac{1}{2}}$ & $\frac{g_{m_{2}} g_{m_{3}}}{C_{2} g_{m_{4}}}$ \\
\hline 5. Fig. 8 & 1 & 1 & $\frac{g_{m_{1}} g_{m_{2}}}{C_{1} C_{2}}$ & {$\left[\frac{g_{m_{1}} C_{2}}{g_{m_{2}} C_{1}}\right]^{\frac{1}{2}}$} & $\frac{g_{m_{2}}}{C_{2}}$ \\
\hline 6. Fig. 9 & $\frac{g_{m_{1}}}{g_{m_{b}}}$ & $\frac{g_{m_{s}}}{g_{m_{s}}}$ & $\frac{g_{m_{1}} g_{m_{2}} g_{m_{4}}}{C_{1} C_{2} g_{m_{\mathrm{s}}}}$ & $\frac{g_{m_{3}}}{g_{m_{3}}}\left[\frac{g_{m_{1}} C_{2} g_{m_{1}}}{g_{m_{2}} C_{1} g_{m_{3}}}\right]^{\frac{1}{2}}$ & $\frac{g_{m_{2}} g_{m_{3}}}{C_{2} g_{m_{5}}}$ \\
\hline
\end{tabular}




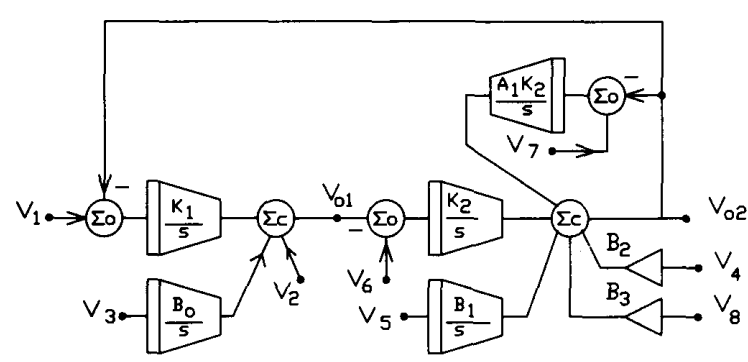

(a)

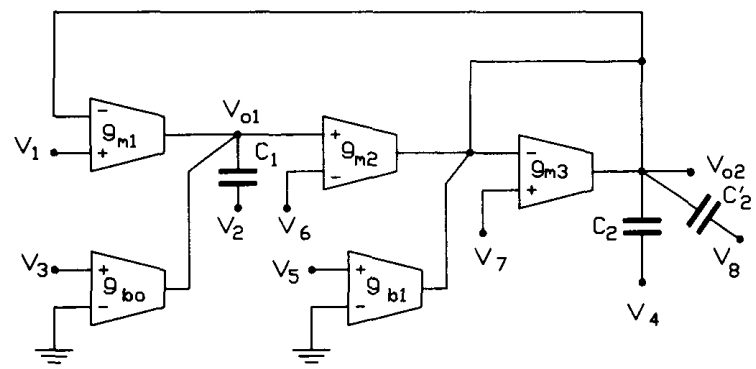

(b) Fig. 10. Filter from Fig. 7. (a) Block diagram. (b) Circuit implementa-

summer. It will be shown later that the outputs of this structure have very desirable properties. Table I shows the mathematical expressions for $\omega_{p}$ and $Q_{p}$ for all the derived structures. Note that for these topologies, except for topology 5 , the $\omega_{p}$ and the $\omega_{p} / Q_{p}$ factor can be independently tuned. Observe that these six structures of Figs. 7-9 represent general families of the two integrator loop OTA filter structures. They can be used as the basis to implement general biquadratic filter structures.

\section{IMPLEMENTATION OF THE Transmission Zeros}

Transmission zeros are obtained by injecting weighted signals into the dead networks (Figs. 7-9). There are two methods of feeding in a signal source without destroying the just established system poles of the dead network:

1) by lifting completely (or partially) any ground connection off ground and connecting it to an input voltage;

2) by feeding an additional current (in our case generated by an OTA) into an impedance node.

To demonstrate the implementation of transmission zeros we will consider in detail three general biquadratic filters.

a) The first second-order filter (Filter A) uses the dead network of Fig. 7 (Topology 2 of Table I) with $A_{0}=1$ and has eight different input signals. The block diagram of the filter is shown in Fig. 10(a) and its corresponding circuit implementation is illustrated in Fig. 10(b). Note that the capacitor $C_{2}$ is ungrounded and split into two capacitors $C_{2}^{\prime}$ and $C_{2}^{\prime}$, and two signal $V_{4}$ and $V_{8}$ are connected to the ungrounded capacitor terminals. ${ }^{3}$ This additional capacitor and input path allows one to obtain a low-pass notch. This technique is only illustrated in this structure, but it can be used with any of the other dead networks discussed before. The output ${ }^{4} V_{o_{2}}$ is given by

$$
\begin{aligned}
V_{o_{2}}=\frac{1}{D_{1}(s)}\{ & K_{1} K_{2} V_{1}+s K_{2} V_{2}+B_{0} K_{2} V_{3}+s^{2} B_{2} V_{4} \\
& \left.+s B_{1} V_{5}-s K_{2} V_{6}+s A_{1} K_{2} V_{7}+s^{2} B_{3} V_{8}\right\}
\end{aligned}
$$

where

$$
D_{1}(s)=s^{2}+A_{1} K_{2} s+K_{1} K_{2}
$$

with $K_{1}=g_{m 1} / C_{1}, B_{0}=g_{b o} / C_{1}, K_{2}=g_{m 2} /\left(C_{2}+C_{2}^{\prime}\right)$, $B_{1}=g_{b 1} /\left(C_{2}+C_{2}^{\prime}\right), A_{1} K_{2}=g_{m 3} /\left(C_{2}+C_{2}^{\prime}\right), B_{2}=C_{2}^{\prime} /$ $\left(C_{2}^{\prime}+C_{2}\right)$, and $B_{3}=C_{2} /\left(C_{2}+C_{2}^{\prime}\right)$. Observe that $B_{0}$ and $B_{1}$ can change their sign by simply interchanging the corresponding OTA input terminals. One particular case is obtained when $g_{m 1}=g_{m 2}=g_{m}, \omega_{p}$ can be adjusted linearly with $g_{m}$, i.e., $\omega_{p}=g_{m} / \sqrt{C_{1}\left(C_{2}+C_{2}^{\prime}\right)}$. Although $Q_{p}$ also changes with $g_{m}$, if used in a second-order bandpass filter, the bandwidth BW becomes independent of $g_{m}$, i.e., BW $=$ $g_{m 3} /\left(C_{2}+C_{2}^{\prime}\right)$. The implementation of $B_{2}$ and $B_{3}$ is easily appreciated for the case of making all the inputs zero, except $V_{8}=V_{1}=V_{\text {in }}$, the corresponding transfer function yields

$$
\begin{aligned}
& H(s)=\frac{V_{o 2}}{V_{\text {in }}}=\frac{s^{2}+\frac{g_{m 1} g_{m 2}}{C_{1} C_{2}}}{s^{2}+s \frac{g_{m 3}}{\left(C_{2}+C_{2}^{\prime}\right)}+\frac{g_{m 1} g_{m 2}}{C_{1}\left(C_{2}+C_{2}^{\prime}\right)}} \\
& \cdot \frac{C_{2}}{C_{2}^{\prime}+C_{2}} \text {. }
\end{aligned}
$$

Equation (10) represents a low-pass notch filter. If the $j \omega$ zeros need not be independently controlled by a transconductance, this circuit could be chosen by the designer.

b) Next we describe a topology (Filter B) with a reduced number of OTA's based on Fig. 8 (Topology 5 of Table I) with $A_{0}=A_{1}=1$. Fig. 11(a) and (b) shows the block diagram and its corresponding circuit implementation, respectively. The output $V_{o 2}$ yields

$$
V_{o 2}=\frac{1}{D_{2}(s)}\left\{K_{1} K_{2} V_{1}+s K_{2} V_{2}+B_{o} K_{2} V_{3}+s^{2} V_{4}+s B_{1} V_{5}\right\}
$$

where

$$
D_{3}(s)=s^{2}+K_{2} s+K_{1} K_{2}
$$

with $K_{1}=g_{m_{1}} / C_{1}, K_{2}=g_{m_{2}} / C_{2}, B_{0}=g_{b_{0}} / C_{1}$, and $B_{1}=$ $g_{b_{1}} / C_{2}$.

c) The Filter $\mathrm{C}$ is based on Fig. 9 (Topology 6 of Table I) and is illustrated in Fig. 12. The block diagram shows 10 input signals. It has 8 OTA's. The output signal $V_{o 2}$ is

${ }^{3}$ This case corresponds to partially lifting the capacitor $C_{2}$ off ground.
${ }^{4}$ The output $V_{O_{2}}$ is chosen as the output of the lossy integrator. 


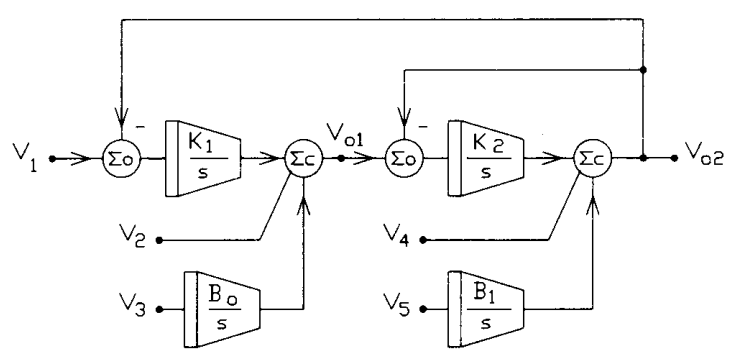

(a)

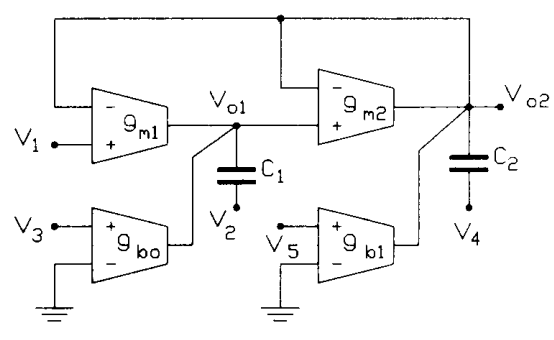

(b)

Fig. 11. Filter B, from Fig. 8 (with $A_{0}=A_{1}=1$ ). (a) Block diagram representation. (b) Circuit implementation.

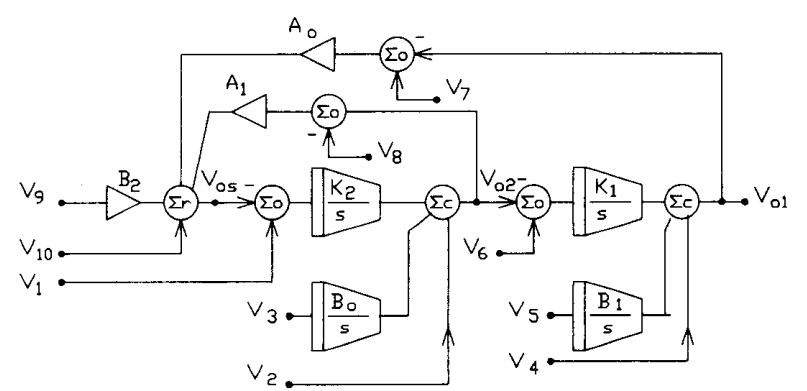

(a)

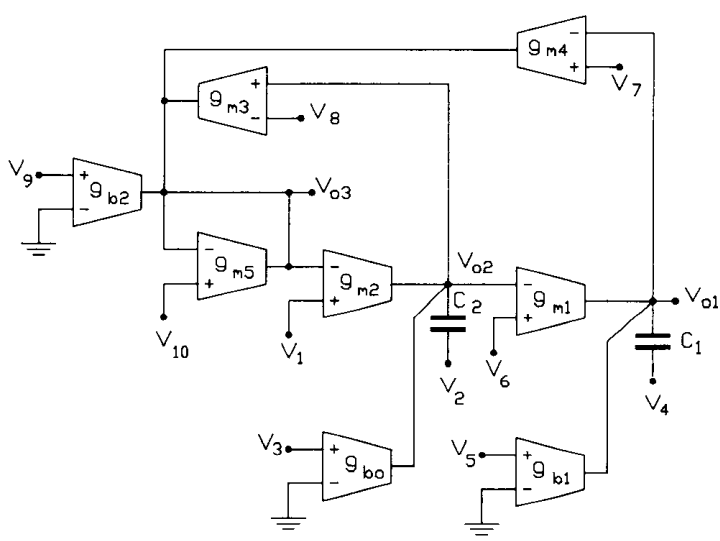

(b)

Fig. 12. Filter C, from Fig. 9. (a) Block diagram representation. (b) Circuit implementation.

given by

$$
\begin{aligned}
V_{o 2}= & \frac{1}{D_{6}(s)}\left\{s K_{2} V_{1}+s^{2} V_{2}+s B_{o} V_{3}+s K_{2} A_{o} V_{4}\right. \\
& +B_{1} A_{o} K_{2} V_{5}+K_{1} K_{2} A_{o} V_{6}-s A_{o} K_{2} V_{7} \\
& \left.+s A_{1} K_{2} V_{8}-s B_{2} K_{2} V_{9}-s K_{2} V_{10}\right\}
\end{aligned}
$$

where

$$
D_{6}(s)=s^{2}+s K_{2} A_{1}+A_{o} K_{1} K_{2} .
$$

Here $K_{1}, K_{2}$, and $B_{1}$, are the same as in Filter B plus, $B_{0}=g_{b 0} / C_{2}, \quad B_{2}=g_{b 2} / g_{m 5}, \quad A_{0}=g_{m 4} / g_{m 5}$, and $A_{1}=$ $g_{m 3} / g_{m 5}$.

\section{Observations on the Structures}

The selection of a particular filter structure depends on a specific application. Next we discuss several particular practical cases.

i) Suppose a fixed low $Q_{p}$ is required and $\omega_{p}$ as well as the zero locations need to be variable. In the case the Filter B of Fig. 11 with 4 OTA's, is very adequate when $V_{1}=V_{2}$ $=0$ and $V_{3}=V_{4}=V_{5}=V_{\text {in }}$, the corresponding transfer function becomes

$$
H(s)=\frac{V_{o 2}}{V_{\text {in }}}=\frac{s^{2}+\frac{g_{b 1}}{C_{2}} s+\frac{g_{b o} g_{m 2}}{C_{1} C_{2}}}{s^{2}+\frac{g_{m 2}}{C_{2}} s+\frac{g_{m 1} g_{m 2}}{C_{1} C_{2}}} .
$$

Note that $g_{b 1}$ can also be implemented with a negative sign of the allpass case. A simple design approach is the following: make $g_{m 1}=g_{m 2}=g_{m}$, then for a given $Q_{p}, \omega_{p}$ and assuming $C_{1}$ is known we obtain $C_{2}=C_{1} Q_{p}^{2}$ and $g_{m}=C_{1} Q_{p} \omega_{p}$. For the particular cases of LP, BP, HP and (all pass); all the inputs are zero except $V_{3}, V_{5}, V_{4}$ and $\left(V_{3}\right.$, $V_{4}$, and $\left.V_{5}\right)$, respectively. The design equation for the zeros become

$$
\begin{aligned}
\text { LP } & g_{b o} & =H_{L P} Q_{p} \omega_{p} C_{1} \\
\text { BP } & g_{b 1} & =H_{B P} Q_{p} \omega_{p} C_{1} \\
\text { HP } & H_{H P} & =1 \\
\text { AP } & g_{b 1} & =-g_{m 2}, g_{b o}=g_{m 1}
\end{aligned}
$$

where $H_{\mathrm{LP}}=H(0), H_{\mathrm{BP}}=H\left(\omega_{p}\right)$, and $H_{\mathrm{HP}}=H(\infty)$. Direct analysis of this filter topology shows that the output signals $V_{1}$ and $V_{02}$, at $\omega_{p}$ differ by $Q_{p}$. This is not so critical for low $Q_{p}$ values. A serious voltage swing problem could be faced for high $Q_{p}$ values, this is discussed in Section V. Furthermore, a large desired $Q_{p}$ cannot be obtained due to 
the nonideal input and output impedances of the OTA's; this will also be demonstrated in Section V.

ii) Now assume the previous design specifications but $Q_{p}$ is required to be variable. A solution can be obtained using the structure of Fig. 10 (Filter A), when $V_{4}=V_{8}=V_{3}$ $=V_{5}=V_{\text {in }}$ and $V_{1}=V_{2}=V_{6}=V_{7}=0$. The resulting transfer function becomes

$$
H(s)=\frac{s^{2}+s \frac{g_{b 1}}{\left(C_{2}+C_{2}^{\prime}\right)}+\frac{g_{b o} g_{m s}}{C_{1}\left(C_{2}^{\prime}+C_{2}\right)}}{s^{2}+s \frac{g_{m 3}}{\left(C_{2}+C_{2}^{\prime}\right)}+\frac{g_{m 1} g_{m 2}}{C_{1}\left(C_{2}+C_{2}^{\prime}\right)}} .
$$

Note that $C_{2}+C_{2}^{\prime}$ can be substituted by a single capacitor, $C_{2}$. Furthermore, assuming $C_{1}$ and $C_{2}$ are known, then the following expressions are obtained:

$$
\begin{aligned}
g_{m 1} g_{m 2} & =C_{1} C_{2} \omega_{p}^{2} \\
g_{m 3} & =\frac{C_{2} \omega_{p}}{Q_{p}} .
\end{aligned}
$$

The maximum voltage swing can be adjusted, depending on the transfer function type. For instance, for the following cases we have

$$
\begin{aligned}
\text { LP: } \quad g_{m 2} & =g_{m 2}=C_{2} \omega_{p} / Q_{p} \\
g_{b o} & =g_{m 1} H_{\mathrm{LP}} \\
g_{m 1} & =\omega_{p} Q_{p} C_{1} \\
\mathrm{BP}: \quad C_{1} & =C_{2}=C \\
g_{m 1} & =g_{m 2}=g_{m}=C \omega p \\
g_{m 3} & =C \omega_{p} / Q_{p} \\
g_{b 1} & =g_{m} H_{\mathrm{BP}} / Q_{p} \\
H_{\mathrm{HP}} & =1 \\
g_{m 2} & =g_{m 3}=C_{2} \omega_{p} / Q_{p} \\
g_{m 1} & =C_{1} \omega_{p} Q_{p}
\end{aligned}
$$

where $H_{\mathrm{LP}}, H_{\mathrm{BP}}$, and $H_{\mathrm{HP}}$ are defined as before.

iii) A filter with three simultaneous outputs is required; these outputs must be high-pass, bandpass, and low-pass types. The structure of Fig. 12 (Filter C) can implement the desired outputs. This is satisfied when all inputs except $V_{9}$ are zero. Then the transfer function yields

$$
\begin{aligned}
& H_{3}(s)=\frac{V_{o 3}}{V_{9}}=\frac{\frac{g_{b 2}}{g_{m 5}} s^{2}}{s^{2}+\frac{g_{m 2} g_{m 3}}{C_{2} g_{m 5}} s+\frac{g_{m 4} g_{m 1} g_{m 2}}{g_{m 5} C_{1} C_{2}}} \\
& H_{2}(s)=\frac{V_{o 2}}{V_{9}}=\frac{-\frac{g_{b s} g_{m 2}}{g_{m 5} C_{2}} s}{s^{2}+\frac{g_{m 2} g_{m 2}}{C_{2} g_{m 5}}+\frac{g_{m 4} g_{m 1} g_{m 2}}{g_{m 5} C_{1} C_{2}}} \\
& H_{1}(s)=\frac{V_{o 1}}{V_{9}}=\frac{\frac{g_{b 2}}{g_{m 5}} \frac{g_{m 1} g_{m 2}}{C_{1} C_{2}}}{s^{2}+\frac{g_{m 2} g_{m 3}}{C_{2} g_{m 5}} s+\frac{g_{m 4} g_{m 1} g_{m 2}}{g_{m 5} C_{1} C_{2}}}
\end{aligned}
$$

A set of simple design equations follows. Given $\omega_{p}, Q_{p}$, $H_{3}(\infty), H_{2}\left(\omega_{p}\right)$, and $H_{1}(0)$ we obtain

$$
\begin{aligned}
\frac{g_{b 2}}{g_{m 5}} & =H_{3}(\infty) \\
\frac{g_{m 2}}{C_{2}} & =\frac{\omega_{p} H_{2}\left(\omega_{p}\right)}{H_{3}(\infty) Q_{p}} \\
\frac{g_{m 1}}{C_{1}} & =\frac{\omega_{p} Q_{p} H_{1}(0)}{H_{2}\left(\omega_{p}\right)} \\
\frac{g_{m 4}}{g_{m 5}} & =\frac{H_{3}(\infty)}{H_{1}(0)} \\
\frac{g_{m 3}}{g_{m 5}} & =\frac{H_{3}(\infty)}{H_{2}\left(\omega_{p}\right)} .
\end{aligned}
$$

Furthermore, observe that the input is not fed through the ungrounded capacitor, which is an additional positive property. That is, a high-pass output does not require the input to be fed through a capacitor. This structure is the homologous to the popular KHN state variable op amp structure.

By selecting the proper inputs and/or outputs of the different structures, many options that satisfy a variety of requirements can be obtained. In some applications the design requirements need to equalize the maximum voltage swing at the OTA outputs to improve the dynamic range. Some structures such as the one shown in Fig. 12 for one particular input allow to adjust the maximum voltage swing at the output of each OTA. However, in some cases the equalization of voltage swings can not be accomplished, this problem is addressed next.

\section{Maximum Voltage Swing Considerations}

Let us consider for illustration purposes the structure of Fig. 10(a) with only one nonzero input $V_{\text {in }}=V_{6}$. The corresponding transfer functions become

$$
H_{1}(s)=\frac{V_{o 1}}{V_{6}}=\frac{K_{1} K_{2}}{s^{2}+s A_{1} K_{2}+K_{1} K_{2}}
$$

and

$$
H_{2}(s)=\frac{V_{o 2}}{V_{6}}=\frac{-s K_{2}}{s^{2}+s A_{1} K_{2}+K_{1} K_{2}} .
$$

Furthermore for $Q_{p}>1$, the maximum magnitudes of the transfer functions occur at $\omega=\omega_{p}$,

$$
\left|H_{1}\left(j \omega_{p}\right)\right|=\frac{K_{1} K_{2}}{\omega_{p} A_{1} K_{2}}=Q_{p}
$$

and

$$
\left|H_{2}\left(j \omega_{p}\right)\right|=\frac{K_{2}}{A_{1} K_{2}}=\frac{K_{2}}{\omega_{p}} Q_{p} .
$$

These maximum magnitudes are equated to $Q_{p}$ if $K_{1}=K_{2}$ $=\omega_{p}$. Equations (22) and (23) for $V_{6}=1 \mathrm{~V}$ show that for practical cases $V_{01}\left(\omega_{p}\right)=Q_{p}$ volts, and $V_{02}\left(\omega_{p}\right) \leqslant Q_{p}$ volts, 


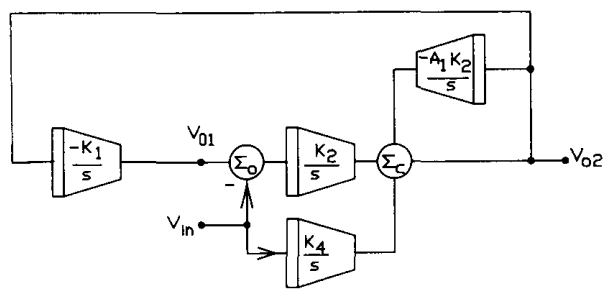

(a)

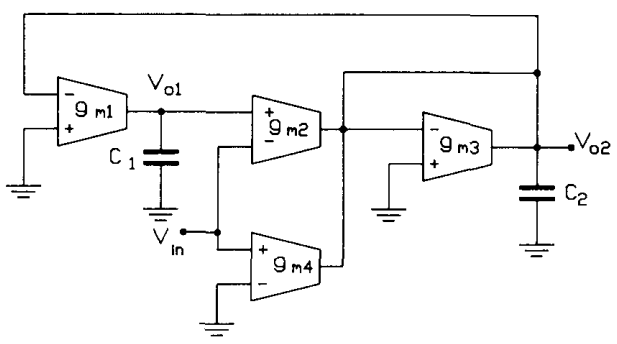

(b)

Fig. 13. Signal nodes voltage equalization. (a) Block diagram representation, (b) A circuit implementation.

this is not a desirable feature for the dynamic range of the filter, especially for high $Q_{p}$ 's. This problem can be compensated by the inclusion input signal attenuation, as done by the additional integrating path in Fig. 13(a). The signal injection becomes proportional to $M=\left(K_{2}-K_{4}\right) / K_{2}$. The modified transfer functions and maximum magnitudes can be obtained by multiplying (20) through (23) by the factor $M$. In particular, we obtain

$$
\begin{aligned}
H_{1}(s) & =\frac{K_{1}\left(K_{2}-K_{4}\right)}{s^{2}+s A_{1} K_{2}+K_{1} K_{2}} \\
& =M \frac{K_{1} K_{2}}{s^{2}+s A_{1} K_{2}+K_{1} K_{2}} \\
H_{2}(s) & =\frac{-s\left(K_{2}-K_{4}\right)}{s^{2}+s A_{1} K_{2}+K_{1} K_{2}} \\
& =M \frac{-s K_{2}}{s^{2}+s A_{1} K_{2}+K_{1} K_{2}}
\end{aligned}
$$

and

$$
H_{1}\left(j \omega_{p}\right)\left|=M Q_{p},\right| H_{2}\left(j \omega_{p}\right) \mid=M \frac{K_{2}}{\omega_{p}} Q_{p} .
$$

Observe that for the original filter the maximum gain of $H_{2}(s)$ was fixed and equal to $Q_{p}$. However, the modified filter can be designed for any $Q_{p}$ and still have $\left|H_{1}\left(j \omega_{p}\right)\right|$ $=\left|H_{2}\left(j \omega_{p}\right)\right|=G<Q_{p}$, where $G$ is an arbitrary practical value. Let $K_{4}=m K_{2}$, and $M=1-m$. Then, given $G, \omega_{p}$, and $Q_{p}$, the design equations using (7) and (26) yield

$$
\begin{aligned}
& K_{1}=K_{2} \\
& A_{1}=1 / Q_{p} \\
& m=1-\frac{G}{Q_{p}} \\
& m=1-\frac{G}{Q_{p}} \\
& K_{4}=m K_{2} .
\end{aligned}
$$

One possible implementation of Fig. 13(a) is shown in Fig. 13(b).

\section{Remarks on Nonidealities of OTA Filters}

The nonideal characteristics of the OTA will limit the performance of the filter structures discussed before. Some of the factors limiting the performance of the OTA filters are the finite input and output impedances and the excess phase of the CMOS OTA. The finite output resistance will affect mainly the lossless integrators at low frequency, the input and output capacitances will affect especially the performance of the summer $\Sigma_{r}$ at high frequency. The summer $\Sigma_{o}$ will cause several distortion for large input voltage differences at the input of the OTA. In general, a $\Sigma_{C}$ summer has better dynamic range than a $\Sigma_{r}$ summer. The excess phase becomes more critical when the number of OTA's increase in the two-integrator loop.

To illustrate the effects of the OTA non-idealities on the filter's frequency performance, assume that each OTA ${ }^{5}$ has a differential input impedance $Z_{\mathrm{di}}=1 / s C_{\mathrm{di}}$, and differential output impedances modeled as the parallel combination of $R_{o i}$ and $C_{o i}$. Let us consider the structure of Fig. 8 with $A_{0}=A_{1}=1$, and the input signal fed at the + terminal of OTA 1. If the output is taken as $V_{o 2}$, the ideal transfer function is

$$
\begin{aligned}
H_{I}(s)=\frac{V_{02}}{V_{i}} & =\frac{\frac{g_{m 1} g_{m 2}}{C_{1} C_{2}}}{s^{2}+\frac{g_{m 2}}{C_{2}} s+\frac{g_{m 1} g_{m 2}}{C_{1} C_{2}}} \\
& =\frac{\omega_{p}^{2}}{s^{2}+\frac{\omega_{p}}{Q_{p}} s+\omega_{p}^{2}} .
\end{aligned}
$$

After the input and output impedance of the two OTA's are considered, (28) yields

$$
H_{r}(s)=H_{\infty} \frac{s^{2}+\frac{\omega_{z}}{Q_{z}} s+\omega_{z}^{2}}{s^{2}+\frac{\omega_{p r}}{Q_{p r}} s+\omega_{p r}^{2}}
$$

\footnotetext{
${ }^{5}$ The characterization of the input and output impedances of the OTA is based on an OTA of the type described in [17], [23].
} 
where

$$
\begin{aligned}
& H_{\infty}=\frac{\frac{C_{d 1}}{C_{2}}\left(1+\frac{C_{o 1}}{1}+\frac{C_{d 2}}{C_{1}}\right)}{1+\frac{C_{o 1}+C_{d 2}}{C_{1}}+\frac{C_{o 2}+C_{d 1}+C_{d 2}}{C_{2}}+\left(\frac{C_{o 1}+C_{d 2}}{C_{1}}\right)\left(\frac{C_{o 2}+C_{d 1}}{C_{2}}\right)+\frac{C_{o 1} C_{d 2}}{C_{1} C_{2}}} \\
& \omega_{p r}=\omega_{p} \sqrt{\frac{1+\frac{1}{A_{v 1}}\left(1+\frac{1}{A_{v 2}}\right)}{1+\frac{C_{o 1}+C_{d 2}}{C_{1}}+\frac{C_{o 2}+C_{d 1}+C_{d 2}}{C_{2}}+\left(\frac{C_{o 2}+C_{d 1}}{C_{2}}\right)+\frac{C_{o 1} C_{d 2}}{C_{1} C_{2}}}} \\
& Q_{p r}=Q_{p} \frac{\sqrt{1+\frac{C_{o 1}+C_{d 2}}{C_{1}}+\frac{C_{o 2}+C_{d 1}+C_{d 2}}{C_{2}}+\left(\frac{C_{o 1}+C_{d 2}}{C_{1}}\right)\left(\frac{C_{o 2}+C_{d 1}}{C_{2}}\right)+\frac{C_{o 1} C_{d 2}}{C_{1} C_{2}}} \cdot \sqrt{1+\frac{1}{A_{v 1}}\left(1+\frac{1}{A_{v 2}}\right)}}{1+\frac{C_{o 1}}{C_{1}}\left(1+\frac{1}{A_{v 2}}\right)+\frac{1}{A_{v 2}}\left(1+\frac{C_{d 2}}{C_{1}}\right)+Q_{p}^{2}\left\{\left(\frac{C_{d 2}}{C_{2}}\left(1+\frac{1}{A_{v 1}}\right)+\frac{1}{A_{v 1}}\left(1+\frac{C_{o 2}+C_{d 1}}{C_{2}}\right)\right\}\right.} \\
& \omega_{z}=\omega_{p} \frac{1}{\sqrt{1+\frac{C_{o 1}+C_{d 2}}{C_{1}}}} \sqrt{\frac{1}{\frac{C_{d 1}}{C_{2}}}} \\
& Q_{z}=\frac{1}{Q_{p}} \frac{1}{\frac{1}{A_{v 1}}+\frac{C_{d 2}}{C_{o 2}}} \sqrt{\frac{1+\frac{C_{o 1}+C_{d 2}}{C_{1}}}{\frac{C_{d 1}}{C_{2}}}}
\end{aligned}
$$

and

$$
A_{v i}=g_{m i} R_{o i}, \quad i=1,2
$$

Note that for an ideal filter all the capacitor ratios in (30) to (34) become zero. It can also be observed that the real transfer function $H_{r}(s)$ differs from the ideal in several aspects. The actual low-frequency gain

$$
\left(H_{\infty} \frac{\omega_{z}^{2}}{\omega_{p r}^{2}}=\frac{1}{1+\frac{1}{A_{V 1}}\left(1+\frac{1}{A_{V 2}}\right)}\right)
$$

can be about $-0.09 \mathrm{~dB}$, for typical values of $A_{v 1}=A_{v 2}=$ 100 , instead of the ideal $0 \mathrm{~dB}$. Furthermore the highfrequency response is not zero, the minimum gain is given by (30) which can be roughly approximated to $C_{d 1} / C_{2}$. We have simulated this structure with SPICE $^{6}$ for an ideal $Q_{p}=10$ and different $f_{p}$ by fixing the transconductance values and selecting $C_{1}$ and $C_{2}$ accordingly. Table II shows the $Q_{p}$ and $f_{p}$ deviations and Table III shows the parasitic zero characteristics. The analytical expressions (29)-(34) compare well with the SPICE results. ${ }^{7}$ These results are also included in Tables II and III. Major discrepancies on

${ }^{6}$ The OTA used is described in [23].

${ }^{7}$ We used $g_{m 1}=56.1 \mu$ mhos, $g_{m 2}=5.61 \mu$ mhos, $C_{d 1}=C_{d 2}=38 \mathrm{fF}$, $R_{o 1}=1.9 \mathrm{M} \Omega, R_{o 2}=19 \mathrm{M} \Omega, C_{o 1}=365 \mathrm{fF}$ and $C_{o 2}=36.5 \mathrm{fF}$ to model the

TABLE II

COMPARISON BETWEen SPICE AND THE ANALYTICAL EQUATIONS (29)-(32) WHERE $\% Q_{p r}=100 \times Q_{p r}-Q_{p} / Q_{p}$, AND HERE $\mathscr{Q}_{0} Q_{p r}=100 \times Q_{p r}-$
$\mathscr{O} f_{p r}=100 \times f_{p r}-f_{p} / f_{p}$

\begin{tabular}{|l|l|l|l|l|}
\cline { 2 - 5 } \multicolumn{1}{c|}{} & \multicolumn{2}{|c|}{ ANALYTICAL } & \multicolumn{2}{c|}{ SPICE } \\
\hline$f_{p}(\mathrm{~Hz})$ & $\% Q_{\mathrm{pr}}$ & $\% f_{\mathrm{pr}}$ & $\% Q_{r}$ & $\% f_{p r}$ \\
\hline $10^{2}$ & -47.6 & 0.455 & -47.4 & 0.242 \\
$10^{3}$ & -47.6 & 0.433 & -47.4 & 0.220 \\
$10^{4}$ & -47.7 & 0.211 & -47.4 & -0.00 \\
$10^{5}$ & -48.7 & -1.94 & -47.3 & -2.00 \\
$10^{8}$ & -56.0 & -18.0 & -47.2 & -18.1 \\
$10^{7}$ & -75.5 & -61.6 & -57.9 & -61.2 \\
\hline
\end{tabular}

TABLE III

COMPARISON BETWEEN SPICE AND THE ANALYTIC EQUATIONS (33)-(34) for the Parasitic Zero Characteristic

\begin{tabular}{|l|l|l|l|l|}
\cline { 2 - 5 } \multicolumn{1}{c|}{} & \multicolumn{2}{|c|}{ ANALYTICAL } & \multicolumn{2}{c|}{ SPICE } \\
\hline$f_{p}(\mathrm{~Hz})$ & $Q_{x}$ & $f_{z}(\mathrm{MHz})$ & $Q_{z}$ & $f_{z}(\mathrm{MHz})$ \\
\hline $10^{2}$ & 152. & 0.153 & 704 & 0.155 \\
$10^{3}$ & 48.0 & 0.485 & 196 & 0.493 \\
$10^{4}$ & 15.2 & 1.53 & 59.4 & 1.56 \\
$10^{5}$ & 4.91 & 4.74 & 20.2 & 4.82 \\
$10^{6}$ & 1.84 & 12.6 & 6.67 & 12.9 \\
$10^{7}$ & 1.15 & 20.2 & 3.49 & 21.2 \\
\hline
\end{tabular}




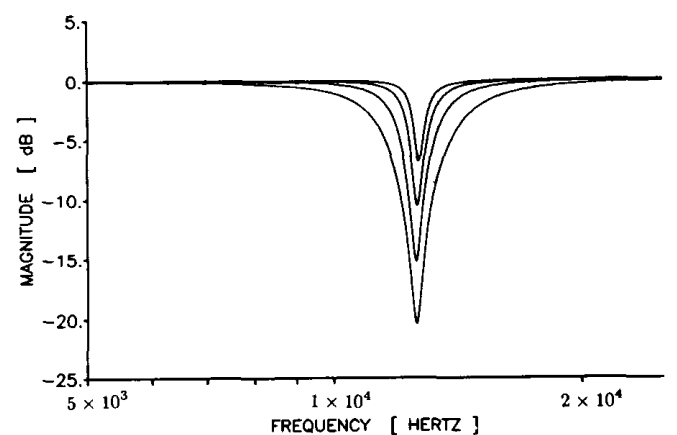

(a)

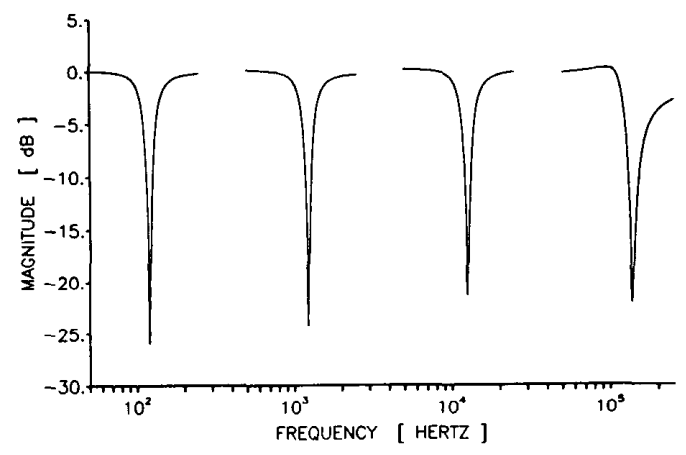

(b)

Fig. 14. Notch filter experimental results. (a) Fixed $f_{p}=12.7 \mathrm{kHz}$ and variable $Q_{p}=\{4,8,16,32\}$. Notch filter experimental results; (b) Fixed $Q_{p}=4$ and variable $f_{p}=\{120,1.22 \mathrm{~K}, 12.52 \mathrm{~K}, 136 \mathrm{Kz}\} \mathrm{Hz}$.

the SPICE results and the analytical results occurred at frequencies higher than $1 \mathrm{MHz}$ where the $g_{m}$ 's become frequency-dependent. This dependence could be modeled, but the resulting analytical expressions might be very awkward. The parasitic zero characteristics are well predicted by the hand calculations. These hand calculations could be improved, at the expense of obtaining large complex expressions, by involving more nonidealities of the OTA, i.e., frequency dependent common mode rejection ratio. Thus the minimal values of $C_{1}$ and $C_{2}$ in comparison with the parasitic capacitances must be determined to minimize the frequency response deviations. In a similar way, whenever it was possible, the $A_{v 1}$ and $A_{v 2}$ must be determined to reduce deviations. For example, if $A_{v 1}=1000$ and $Q_{p}=10$, then $\% Q_{p r}$ becomes about 9 percent instead of nearly 48 percent, as shown in Table II. For this filter it should be concluded that small $\% Q_{r}$ can only be obtained for small $Q_{p}$ 's and/or very large $A_{v 1}$.

\section{EXPERIMENTAL RESULTS}

Three breadboard filter examples are provided in this section to show the programmability features of these filters. Commercial OTA LM 3080 type and polyester capacitors $C_{1}=C_{2}=31.8 \mathrm{nF}$ are used for the three examples.

i) Notch Filter: This filter was implemented using the structure of Fig. 10. The experimental results for variable $Q_{p}$ and fixed $f_{p}$ are shown in Fig. 14. The pole frequency

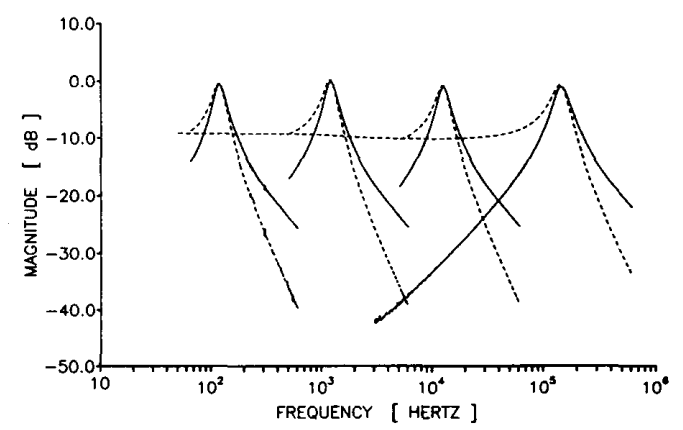

Fig. 15. Maximum amplitude equalization; plots for variable $f_{p}$ and fixed $Q_{p}=8$.

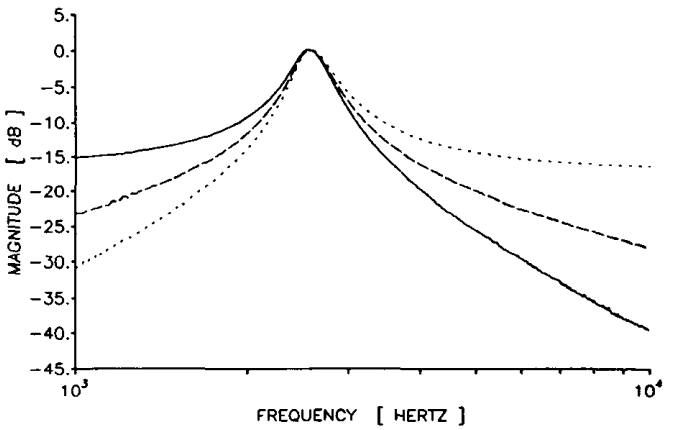

Fig. 16. Low pass, bandpass, and high-pass outputs of a KHN OTA filter, from Fig. 12 with $V_{8}=V_{\text {in }}$

$f_{p}=12.7 \mathrm{kHz}$ was determined with the bias currents of the OTA's 1 and 2, i.e., $I_{\mathrm{BIAS}_{1}}=I_{\mathrm{BLAS}_{2}}=100 \mu \mathrm{A}$. The $Q_{p}$ values were varied via the OTA 3 , for $Q_{p}=\{4,8,16,32\}$ which correspond to $I_{\text {BIAS }_{3}}=\{25,12.5,6.25,3.12\} \mu \mathrm{A}$ and the notch attenuation $=\{19.11,14.11,9.55,5.9\} \mathrm{dB}$. The noise flow was below $58 \mathrm{~dB}(100-\mathrm{Hz}$ bandwidth). Fig. 14(b) shows the experimental results for constant $Q_{p}=4$ and variable $f_{p}$. The summarized results follow:

\begin{tabular}{lcccc}
\hline$f_{p}=f_{z}$ & $(\mathrm{~Hz})$ & 120 & $1.22 \mathrm{~K}$ & $12.52 \mathrm{~K}$ \\
Attenuation & $\mathrm{dB}$ & 29.6 & 24.5 & 21.4 \\
\hline
\end{tabular}

For $f_{z}=136 \mathrm{kHz}$ the corresponding pole frequency, $f_{p}$, and attenuation were $95.8 \mathrm{kHz}$ and $22.2 \mathrm{~dB}$, respectively.

ii) Bandpass Filter: The structure of Fig. 13 was used to implement a bandpass filter. Fig. 15 shows the experimental results for variable $\omega_{p}$ and fixed $Q_{p}=8$. The two OTA outputs (bandpass and low pass) were scaled to have the same peak value, this is also shown in Fig. 15 with the solid and broken lines. The noise level was below $45 \mathrm{~dB}$ for a $100-\mathrm{Hz}$ bandwidth. The $f_{p}$ 's obtained were $\{117,1.21$ $\mathrm{K}, 12.7 \mathrm{~K}, 142 \mathrm{~K}\} \mathrm{Hz}$ and the corresponding peak values were $\{-0.05,-0.4,-1.8\} \mathrm{dB}$. The small deviations of $f_{p}$ and the peak values are due to the nonidealities of the integrators.

iii) KHN OTA Structure: The structure of Fig. 12 was breadboarded with all the input zero except $V_{9}=V_{\text {in }}$. Using the design equations (19) for an equal maximum voltage 
gain of $0 \mathrm{~dB}$ the experimental results of Fig. 16 were obtained. The $\omega_{p}$ and $Q_{p}$ are $25.5 \mathrm{kHz}$ and 8, respectively. Fig. 16 also shows simultaneously the three available outputs of the filter. The noise floor was below $58 \mathrm{~dB}$ for $100-\mathrm{Hz}$ bandwidth.

\section{CONCLUSIONS}

A generation of two integrator loop OTA filters has been presented. The advantages and some practical limitations of these filters have been discussed. Further research on practical frequency limitations on the OTA-C structures here generated is needed. It was indicated that the selection of a particular filter structure of a function of the design specifications. The TAC structures containing only C's and OTA's are very appropriate for IC implementations. Furthermore, fully differential OTA filter structures can be relatively easy to obtain from many of the single ended structures here generated. The generated structures like any other continuous time, require an automatic tuning system to control the voltage dependent time constant. The OTA-C filter structures here discussed, together with flexible and practical CMOS OTA can be a good combination for the realization of practical monolithic continuoustime filters, particularly at high frequencies [21]-[23].

\section{REFERENCES}

[1] C.-C. Park and R. Schaumann, "A high-frequency CMOS linear transconductance element," IEEE Trans. Circuits Syst., vol. CAS 33, pp. 1132-138, Nov. 1986.

[2] K. W. Moulding, "Fully integrated selectivity at high frequency using gyrators," IEEE Trans. Broadcast. Telev. Reg., vol. BTR-19, pp. 176-179, Aug. 1973.

[3] K. S. Tan and P. R. Gray, "Fully integrated analog filters using bipolar JFET technology," IEEE J. Solid-State Circuits, vol. SC-12, pp. 814-821, Dec. 1978 .

[4] K. Fukahor, "A A bipolar voltage-controlled tunable filter," IEEE J. Solid-State Circuits, vol. SC-16, pp. 729-737, Dec. 1981.

[5] J. O. Voorman, W. H. A. Brüls, and J. P. Bawrth, "Integration of analog filters in a bipolar process," IEEE J. Solid-State Circuits vol. SC-17, pp. 713-722, Aug. 1982 .

[6] K. W. Moulding and P. J. Ranking, "Experience with highfrequency gyrator filters including a new video delay-line IC," in Proc. 6th European Conf. on Circuit Theory and Design, pp. 95-98, Sept. 1983.

[7] J. O. Voorman, W. H. A. Brüls, and P. J. Barth, "Bipolar integration of analog gyrator and Laguerre type filters (transconductancecapacitor filters)," in Proc. 6th European Conf. on Circuit Theory and Design, pp. 108-110, Sept. 1983.

[8] G. Tröster and W. Langheinrich, "Monolithic continuous-time analogue filters in NMOS technology," in Proc. IEEE/ISCAS, vol. 3, pp. 1498-1501, May 1984.

[9] H. Khorramabadi and P. R. Gray, "High frequency CMOS continuous-time filters," in Proc. IEEE/ISCAS, vol. 3, pp. 1498-1501, May 1984.

[10] Y. Tsividis, M. Banu, and J. Khoury, "Continuous-time MOSFET-C filters in VLSI," IEEE J. Solid-State Circuits, vol. SC-21, pp. 15-30, Feb. 1986

[11] H. A. Wittlinger, "Applications of the CA3080 an dCA30380A high performance operational transconductance amplifiers," RCA Appl Note ICA-6668

[12] C. F. Wheatley and H. A. Wittlinger, "OTA obsoletes OP.AMP," in P. Nat. Econ. Conf., pp. 152-157, Dec. 1969.

[13] RCA Electronic Components, Linear Integrated Circuits, Model CA3060: Data File 404, Mar. 1970.

[14] RCA Solid-State Division, Data Book, Linear Integrated Circuits, File No. 475, Mar. 1975

[15] M. Bialko and R. W. Newcomb, "Generation of all finite linear circuits using the integrated DVCCS," IEEE Trans. Circuit Theory, vol. CT-18, pp. 733-736, Nov. 1971 .

[16] K. D. Peterson and R. L. Geiger, "CMOS OTA structures with improved linearity," in Proc. 27th Midwest Symp. of Circuits and Systems, pp. 63-66, June 1984.
[17] A. Nedungadi and T. R. Viswanathan, "Design of linear CMOS transconductance elements," IEEE Trans. Circuits Syst., vol. CAS 31, pp. 891-894, Oct. 1984.

[18] R. R. Torrance, T. R. Viswanathjan, and J. V. Hanson, "CMOS voltage to current transconductors," IEEE Trans. Circuits Syst., vol. CAS-32, pp. 1097-1104, Nov. 1985.

[19] H. S. Malvar, "Electronically controlled active-C filter and equalizers with operational transconductance amplifiers," IEEE Trans. Circuits Syst., vol. CAS-31, pp. 645-649, July 1984

[20] R. L. Geiger and E. Sánchez-Sinencio, "Active filter design using operational transconductance amplifiers; A tutorial," IEEE Circuits and Devices Mag., vol. 1, pp. 20-32, Mar. 1985.

[21] S. Masuda and Y. Kitamura, "Design consideration of monolithic continuous time filters," in Proc. IEEE/ISCAS, vol. 3, pp. continuous time filters," in Proc.

[22] C. Plett, M. A. Copeland and R. A. Hadaway, "Continuous-time filters using open-loop tunable transconductance amplifiers," in Proc. IEEE/ISCAS, vol. 3, pp. 1172-1176, San Jose, CA, May 1986. [23] A. Nedungadi and R. L. Geiger, "High frequency voltage con-
trolled continuous time low-pass filter using linearised CMOS integrators," Electron. Lett., vol. 22, pp. 729-731, June 1986.

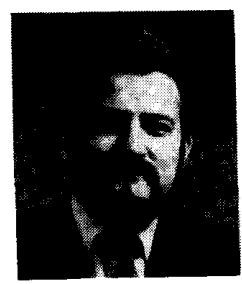

Edgar Sánchez-Sinencio (S'72-M'74-SM'83) received the M.S.E.E. degree from Stanford University, CA, in 1970, and the Ph.D. degree from the University of Illinois, Champaign-Urbana, in 1973. His doctoral thesis was on computer-aided design of microwave integrated circuits.

In 1974, he held an industrial Post-Doctoral position with the Central Research Laboratories, Nippon Electric Company Ltd., Kawasaki, Japan. From 1976 to 1983, he was the Head of the Department of Electronics at the Instituto Nacional de Astrofísica, Optica y Electrónica, Puebla, Mexico. He was a visiting professor in the Department of Electrical Engineering at Texas A\&M University during the academic years of 1979-1980 and 1983-1984, where he is currently a Professor. He was the General Chairman of the 1983 26th Midwest Symposium on Circuits and Systems, and is currently serving as an associated editor of news and events for the IEEE Circuits and Devices Magazine. He was an associate editor of the IEEE TRANSACTIONS ON CiRCUITS AND SYsTEMS from 1985 to 1987 . He is the co-author of the book, Switched-Capacitor Circuits (Van Nostrand-Reinhold, 1984) His current research interest include SC high-frequency circuits, operational transconductance amplifiers applications and computer-aided design of solid-state circuits.

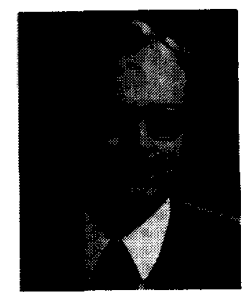

Randall L. Geiger (S'75-M'77-SM'82) received the B.S. degree in electrical engineering and the M.S. degree in mathematics from the University of Nebraska, Lincoln, in 1972 and 1973, respectively, and the Ph.D. degree in electrical engineering from Colorado State University, Fort Collins, in 1977.

Dr. Geiger currently holds the rank of Professor in the Department of Electrical Engineering at Texas A\&M University. He received the Myril B. Reed Best Paper Award at the 1981 Midwest Symposium on Circuits and Systems for presentation of a paper titled "Switched Resistor Filters". $\mathrm{He}$ is co-founder and past president (1982-85) of World Instruments Inc., a firm specializing in microprocessor-based instrumentation.

He served as Conference Chairman at the 1983 UGIM Conference, has been a member of the Midwest Symposium Steering Committee since 
1980 and is a Registered Professional Engineer in the State of Texas. He was an Associate Editor of the IEEE TRANSAcrions on CIRcuITs AND SYSTEMS from 1983 to 1985 , and is currently a Member of the Administrative Committee of the IEEE Circuits and Systems Society and the Circuits and Systems Society Editor of the IEEE CIRCuITS AND DEvices MAGAZINE

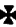

Horacio Nevarez-Lozano received the B.E. degree in industrial electronics engineering from the Regional Institute of Technology of Chihuahua
(ITRCH), Chihuahua, Mexico in 1977, and the M.S. degree in semiconductors and solid state devices from the National Astrophysics, Optics and Electronics Institute (INAOE) of Tonantziutla, Puebla, Mexico in 1980. He is working towards the Ph.D. degree in electrical engineering a Texas A\&M University, College Station, TX.

His technical research interests include analog and digital circuit design, both monolithic and PCB; the physics, modeling and manufacturing of semiconductor devices. His technical activities have involved the design of fiber-optic communication systems for transmission of analog signals and the design of microprocessor-based systems for industrial and instrumentation applications. 